Review Article

\title{
Pulmonary tuberculosis diagnosis, differentiation and disease management: A review of radiomics applications
}

\author{
Tamarisk DU PLESSIS ${ }^{a,}$, , William lan Duncombe RAE ${ }^{b}$, Mike Michael SATHEKGE ${ }^{a}$ \\ ${ }^{a}$ Department of Nuclear Medicine, University of Pretoria, South Africa \\ ${ }^{b}$ Discipline of Medical Imaging Sciences, University of Sydney, Australia \\ *E-mail address: tamarisk.duplessis@gmail.com
}

\begin{abstract}
Pulmonary tuberculosis is a worldwide epidemic that can only be fought effectively with early and accurate diagnosis and proper disease management. The means of diagnosis and disease management should be easily accessible, cost effective and be readily available in the high tuberculosis burdened countries where it is most needed. Fortunately, the fast development of computer science in recent years has ensured that medical images can accurately be quantified. Radiomics is one such tool that can be used to quantify medical images. This review article focuses on the literature currently available on the application of radiomics explicitly for the purpose of diagnosis, differentiation from other pulmonary diseases and disease management of pulmonary tuberculosis. Despite using a formal search strategy, only five articles could be found on the application of radiomics to pulmonary tuberculosis. In all five articles reviewed, radiomic feature extraction was successfully used to quantify digital medical images for the purpose of comparing, or differentiating, pulmonary tuberculosis from other pulmonary diseases. This demonstrates that the use of radiomics for the purpose of tuberculosis disease management and diagnosis remains a valuable data mining opportunity not yet realised.
\end{abstract}

Key words: feature extraction; pulmonary tuberculosis; radiomics.

\section{Introduction}

Pulmonary tuberculosis (PTB) is an ongoing global epidemic and has been identified as a research priority by the World Health Organization (WHO) and various countries' national health departments. ${ }^{1}$ Early and accurate diagnosis and proper disease management is key to fighting this epidemic. To diagnose and manage PTB clinicians generally use a combination of biological methods (such as the Mantoux tuberculin skin test, tuberculosis (TB) blood test or sputum smear tests), ${ }^{2}$ demographic data and radiological methods. ${ }^{3-4}$ Biological methods are used as PTB biomarkers, with a sputum smear culture conversion (smear-positive to smear-negative status) currently the most widely accepted biomarker to predict a relapse-free cure of PTB. ${ }^{5}$ A meta-analysis study showed that this is not a reliable biomarker with a sensitivity of only $40 \%$ (95\% CI 25-56) and specificity of $85 \%$ (95\% CI 77-91) for predicting relapse. ${ }^{5}$ Researchers, therefore, still face the challenge of identifying more sensitive and specific biomarkers that can be used to evaluate TB disease progression and response to treatment quantitively. ${ }^{5-7}$ Another challenge in fighting this global epidemic is the burden of multi-drug resistant TB, where India (27\%), China (14\%) and The Russian Federation (8\%) carry the highest number of cases globally. ${ }^{1}$ To ensure a reduction in incidences and deaths, this epidemic needs to be addressed universally.

The top 8 highest TB burdened countries, who account for two-thirds of the global TB incidence, are all developing countries, with $44 \%$ of all global cases in the South-East Asian region. ${ }^{1}$ In these countries chest x-ray (CXR) remains the most common radiological imaging modality for PTB screening, as access to more sophisticated three-dimensional (3D) imaging modalities are limited ${ }^{8-9}$ Even in countries where patients have easier access to 3D modalities, it was shown that CXR remains the foundation for imaging certain radiological expressions of PTB, e.g. parenchymal disease. ${ }^{10}$ But CT is more sensitive in detecting many other radiological expressions, e.g. lymphadenopathy and early bronchogenic spread in postprimary TB. ${ }^{10}$ Not only is CXR the most widely accessible imaging modality, ${ }^{11}$ but radiation doses to the patients are kept to a minimum by using projection imaging. ${ }^{12}$ Radiation dose and long term radiation effects become a considering factor when screening large cohorts or when multiple follow-up images are acquired. Research has showed that CXR is a very effective diagnostic modality as it yields high sensitivity $(0.78,95 \% \mathrm{CI}$ $0.73-0.82$ ) and moderate to high specificity $(75.7 \%),{ }^{8,13}$ But to utilise these properties expert readers are needed to interpret 
these images, and these experts are often scarce in resourcelimited countries. ${ }^{3}$

With the fast development of computer science in recent years, various research studies have been conducted on the use of artificial intelligence (AI) applications (Machine Learning (ML) and Deep Learning (DL)) to assist with, among other things, medical image processing and interpretation. ${ }^{11} \mathrm{ML}$ is a subset of AI that uses statistical algorithms that enable machines to improve with experience, while DL is a subset of ML and is based on artificial neural networks that enable algorithms to train themselves. Lately, ML and DL have allowed radiological image interpretation to evolve from subjective reporting to objective science, ${ }^{\mathbf{1 1}}$ and from a primary diagnostic tool to a central role player in personalised precision medicine. ${ }^{\mathbf{1 4}}$

PTB causes a wide variety of pathological changes in the lungs which lead to many different radiological manifestations, and AI systems that are developed must have the ability to adapt to and manage these distinct morphological patterns. ${ }^{15}$ Pathological changes visible on CXR include changes in the lung shape, size, and context (texture), which influence the lung-field symmetry. ${ }^{9}$ When radiologists perform bi-lateral comparisons on CXR, the differences in corresponding regions between the left and right lung greatly assist in detecting these abnormalities. ${ }^{\mathbf{1 6}}$ This same principle is mimicked when automatic TB detection systems use feature vectors of various sizes and combinations,, ${ }^{\mathbf{9}-18}$ with different classification algorithms to extract information from radiological images., ${ }^{\mathbf{9} 19}$

To date AI applications are mainly used for the diagnosis of $\mathrm{TB}$, but a few other studies have been done, for example on the automatic differentiation of drug-sensitive TB from drugresistant TB from CXR. ${ }^{19}$ This study tested various classifiers by using them in combination with a set of shape and texture features. It achieved the best performance, with an area under the receiver operating characteristic curve (AUC) of $66 \%$, when using a traditional artificial network (ANN). ${ }^{19}$ Many studies have also been done on differentiating PTB from other pulmonary diseases, often lung cancer, using both CXR and CT scans. The reason for this is that PTB mimics various other pulmonary diseases, and diagnostic imaging of PTB remains challenging. ${ }^{10}$

Automatic PTB screening systems are extremely useful, efficient and can be a low-cost mass screening tool that can be well utilised in resource-constrained countries. ${ }^{20}$ DL methods for some diseases do not yet perform to the same accuracy as radiologists, but it has been demonstrated that automatic PTB detection from $\mathrm{CXR}$ has reached radiologist level performance. ${ }^{\mathbf{2 1}}$ However, research into automated radiological image analysis for disease management and prognosis remains limited. ${ }^{21}$ For quantitative disease management, the automatic algorithms need to produce measurable outputs. One such method to quantify medical images is by using radiomic feature extraction. Although recently developed DL networks have led to more robust models for radiomics, and this has allowed the high-throughput extraction of quantitative features from radiological images, this has not been widely utilised as quantitative outputs need to be extracted from the networks and are generally hidden within the layers of the DL network or within the last layer of the algorithm. ${ }^{22}$

Radiomics is based on the hypothesis that the correct combination of these features, together with the clinical data, can identify significant tissue properties useful in the management of a disease. ${ }^{23}$ Radiomics differs from traditional Computer-aided-detection (CAD) systems in the sense that CAD systems were designed only to diagnose or detect a disease. ${ }^{24}$ Radiomic feature extraction can also be used to quantify disease characteristics and progression from medical images, as it makes use of statistically based imaging analysis algorithms to act as quantitative biomarkers for the identification of radiological features. ${ }^{\mathbf{2 4}-25}$ These can be used to quantify change and categorisation and not only identify and categorise predicted outcomes or disease states. This feature extraction tool allows medical images to be converted into minable multidimensional statistical data sets which characterise the relationship between the high dimensional data of the images. ${ }^{24}$ Radiomics is a complex, multi-step field of study that includes the following identifiable steps: image acquisition, image segmentation, feature extraction and qualification, analysis and database development. ${ }^{25}$ Database development includes developing a radiomics signature or nomogram. A radiomics signature is a computational model built to meet specific clinical needs, ${ }^{\mathbf{2 6}}$ whereas a radiomics nomogram integrates a radiomics signature with the clinical data to evaluate parameters such as prognosis or disease management. ${ }^{27}$ To develop accurate, robust and reproducible radiomics signatures, the knowledge and skills of qualified and experienced researchers are crucial. They need to understand the influence of exposure parameters, image preand post-processing, image segmentation and mask modelling. Experienced researchers also play a vital role in optimisation and standardisation of image acquisition protocols, modelling, developing algorithms and the statistical analysis of high dimensional data.

Extracted features can be sub-categorised into first-order statistical features, shape-based features (morphological 2D and 3D), textural features (or second-order statistical features) and higher-order statistical features. ${ }^{\mathbf{2 8}}$ Higher-order statistics are obtained by applying filters or mathematical transforms to the image before applying statistical algorithms. ${ }^{28}$ Each one of the steps in radiomics poses its own set of challenges. Rizzo et al. explained it well in a narrative review article; "Radiomics: the facts and the challenges of image analysis". ${ }^{23}$

Radiomics had its origin in the medical field of oncology, ${ }^{24}$ and numerous articles have been published on the use of radiomics in tumours, but very few on the application in nonneoplastic diseases such as TB. ${ }^{29}$ The purpose of this review article is to determine what research has already been done on the application of radiomics explicitly for the diagnosis and management of PTB or the differentiation of PTB from other pulmonary diseases. This will also reveal areas not yet addressed 
in the available literature and the potential opportunities for future research. Even though this is a narrative review, a formal systematic research strategy was followed to ensure that all published journal articles on this topic were included.

\section{Methods}

\section{Review search strategy}

A comprehensive search for relevant literature was done by two independent information specialists at two separate universities. The following databases were included in the search: PubMed (US National Library of Medicine), OVID Medline, Scopus, OVID Embase, CINAHL and IEEE Xplore.

The search strategy that was applied to text-words can be summarised as follows: ((radiomics OR radiomic*) OR ('imaging biomarker*' OR 'radiological biomarker*' OR 'texture feature*' OR 'texture analysis')) AND (tuberculosis OR TB). The year limit that was used was 2000 to May 2021 (week 2) when the search was concluded. The term 'radiomics' was first introduced in medicine in $2012,{ }^{30}$ and the year 2000 was an arbitrarily selected date prior to 2012 .

\section{Study selection criteria}

All journal articles were considered, but only studies that met the following inclusion criteria were included: 1) Original studies with full-text articles published in English. 2) Studies with a human study population who were diagnosed with pulmonary tuberculosis. 3) Articles that included medical images (from any imaging modality: CT, MRI, PET/CT and Xrays) as datasets. 4) Studies that used radiomics or texture feature extraction methods. 5) Articles with the aim to develop imaging- or radiological biomarkers for PTB.

Exclusion criteria: 1) Articles that did not extract quantitative information from medical images. 2) Non-peer-reviewed academic journal articles, including conference proceedings.

\section{Search results and data extraction}

The search strategies returned a total of 66 journal articles $(n=$ 18 in the PubMed database, $\mathrm{n}=16$ in the OVID Medline database, $n=5$ in Scopus database, $n=6$ in OVID Embase, $n=$ 5 in CINAHL and $n=16$ IEEE Xplore database). After duplicates were removed, $n=49$ remained. Since a systematic review search strategy was followed, the search results were recorded and summarised accordingly in the PRISMA (Preferred Reporting Items for Systematic Reviews and MetaAnalysis) flow diagram (Figure 1). ${ }^{31}$

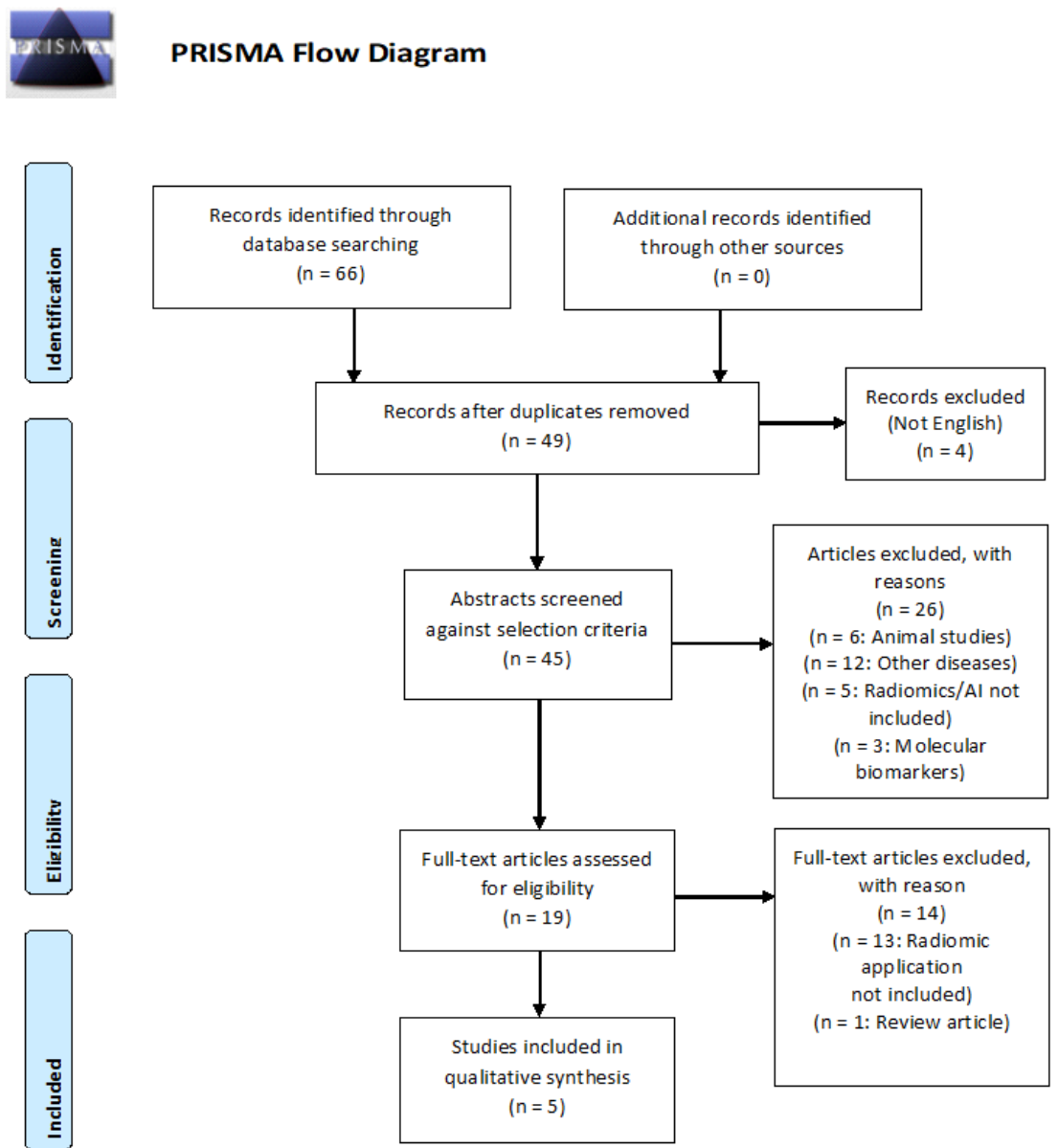

Figure 1. Preferred Reporting Items for Systematic Reviews and Meta-Analysis (PRISMA) flow diagram for journal article screening and selection 


\section{Results}

Despite the thorough search strategy that was followed to ensure complete inclusion of all available published data, only 5 articles could be found that met all the inclusion criteria on the application of radiomics to PTB. The relevant detail for each of the studies, such as radiomics libraries, models, algorithms, dimensionality reduction methods, clinical features, etc. used, is summarised in Table 1.

Study A: In the first article by Bei et al. [2019] radiomic features extracted from CT images were used to create a radiomics signature capable of distinguishing primary progressive PTB from community-acquired pneumonia in children. ${ }^{29}$ Manual segmentation was performed by a radiologist with more than 10 years of experience and verified by a second radiologist. ${ }^{29}$ The radiologist delineated the margins of pulmonary consolidation as the first region-of-interest (ROI) and the mediastinal lymph nodes as the second ROI. ${ }^{29}$ In this study, they developed two radiomics signatures, one from each ROI, and constructed a radiomics model by combining these two signatures. ${ }^{29}$ Finally, they used the least shrinkage and selection operator (LASSO) algorithm to build a predictive nomogram by combining the radiomics model with the clinical data. ${ }^{29}$ The predictive nomogram's classification outperformed the senior radiologist's clinical judgement $(\mathrm{AUC}=0.971,95 \% \mathrm{CI}$ : 0.9121 vs. $\mathrm{AUC}=0.832,95 \%$ CI: $0.677-0.987) .{ }^{29}$

Study B: In the second article Shi et al. [2019], used radiomic features to assist with the identification of opportunistic pulmonary infections (OPIs) misdiagnosed as lung cancers in patients with human immunodeficiency virus (HIV). ${ }^{32}$ In this study $76.2 \%$ of the OPI cohort had PTB and $23.8 \%$ other pulmonary infections. ${ }^{32}$ Semi-automatic segmentation of the lesion was done using in-house software. ${ }^{32}$ The ROI borders were then manually adjusted by a radiologist to ensure that the lesion boundary was entirely included and that the bronchi and vessels were excluded. ${ }^{32}$ The morphological CT features, clinical data and radiomic features were statistically compared between the two disease groups. They concluded that radiomics might assist with the identification of OPIs mimicking lung cancers for central-type lesions. Four radiomic features in these lesions were significantly different (large dependence high gray level emphasis (LDHGLE) $(\mathrm{P}=0.008)$, skewness $(\mathrm{P}=0.017)$, inverse difference normalised (IDN) $(\mathrm{P}=0.017)$ and kurtosis $(P=0.017) .{ }^{32}$ But they found that radiomics features of the peripheral-type lesions might not be useful for differentiating the diseases. ${ }^{32}$

Study C: In the third article Feng et al. [2020] used radiomic features to differentiate between lung tuberculoma and adenocarcinoma presenting as solitary pulmonary solid nodules. ${ }^{33}$ They used a U-net-based volume-of-interest (VOI) segmentation method to automatically delineate the lesions' boundary ${ }^{33} \mathrm{~A}$ radiomics signature consisting of 6 features was identified and combined with clinical data to build a predictive radiomics nomogram using LASSO logistic regression. ${ }^{33}$ The signature showed improved diagnostic accuracy compared to any single model (AUC $=0.9064,95 \%$ CI: 0.9390-0.9931). ${ }^{33}$

Study D: In this article, Cui et al. [2020] developed a radiomics nomogram model, using LASSO algorithms to differentiate TB from lung cancer from preoperative lung CT data. ${ }^{34}$ They have also evaluated different radial dilation distances outside the lesion to determine the best performance. ${ }^{34}$ The nomogram showed good discriminative performance in distinguishing $\mathrm{TB}$ from lung cancer $\quad(\mathrm{AUC}=0.914$, sensitivity $=0.788, \quad$ specificity $=0.907) .{ }^{34}$ This quantitative study again shows improved detection and discrimination performance of medical images when using the radiomics nomogram compared to decisions made by radiologists alone. ${ }^{34}$

Study E: In the final article, Du et al. [2021] developed CT, $\mathrm{PET}$ and $\mathrm{PET} / \mathrm{CT}$ radiomics signatures. These signatures were combined with semantic features to develop radiomics nomograms to differentiate between active pulmonary TB and lung cancer. ${ }^{35}$ Nine CT-based semantic features (maximal tumor diameter, tumor location, cavitation, vacuole, spiculation, vessel convergence, lobulation, pleural indentation and air bronchogram) and two PET-based semantic features (radionecrosis and metabolic activity greater that the adjacent mediastinal blood pool) were included. ${ }^{35}$ They showed that the performance of the CT signature was superior to that of the PET signature $(\mathrm{AUC}=0.86$ vs. $0.79, \mathrm{p}=0.1585)$, and that the $\mathrm{PET} / \mathrm{CT}$ signature improved diagnostic performance even further compared to CT alone (AUC $=0.91$ vs. 0.86, $\mathrm{p}=0.0247){ }^{35}$ They concluded that PET and CT radiomic features could offer complementary diagnostic value when used in combination with the semantic features defined by radiologists. ${ }^{35}$

\section{Discussion}

In this review study, only five articles could be identified that met the inclusion criteria. In all 5 articles, radiomic feature extraction was successfully used to quantify images to compare or differentiate pulmonary tuberculosis from other pulmonary diseases (pneumonia ${ }^{29}$, lung cancers ${ }^{32,34-35}$ and adenocarcinomas $^{33}$ ). All imaging modalities (CT, MRI, Molecular imaging and Planar imaging) were included in the search, but only CT and PET/CT (study E only) scans were used for feature extraction. It is also interesting to note that all five studies were carried out in China. This might be because China is the third highest TB burdened country (accounting for $8.4 \%$ of all global instances), ${ }^{1}$ they are technologically very advanced and most patients have access to $3 \mathrm{D}$ imaging resources. 
Table 1. Summary table that includes comparative, relevant information for the three studies reviewed in this article.

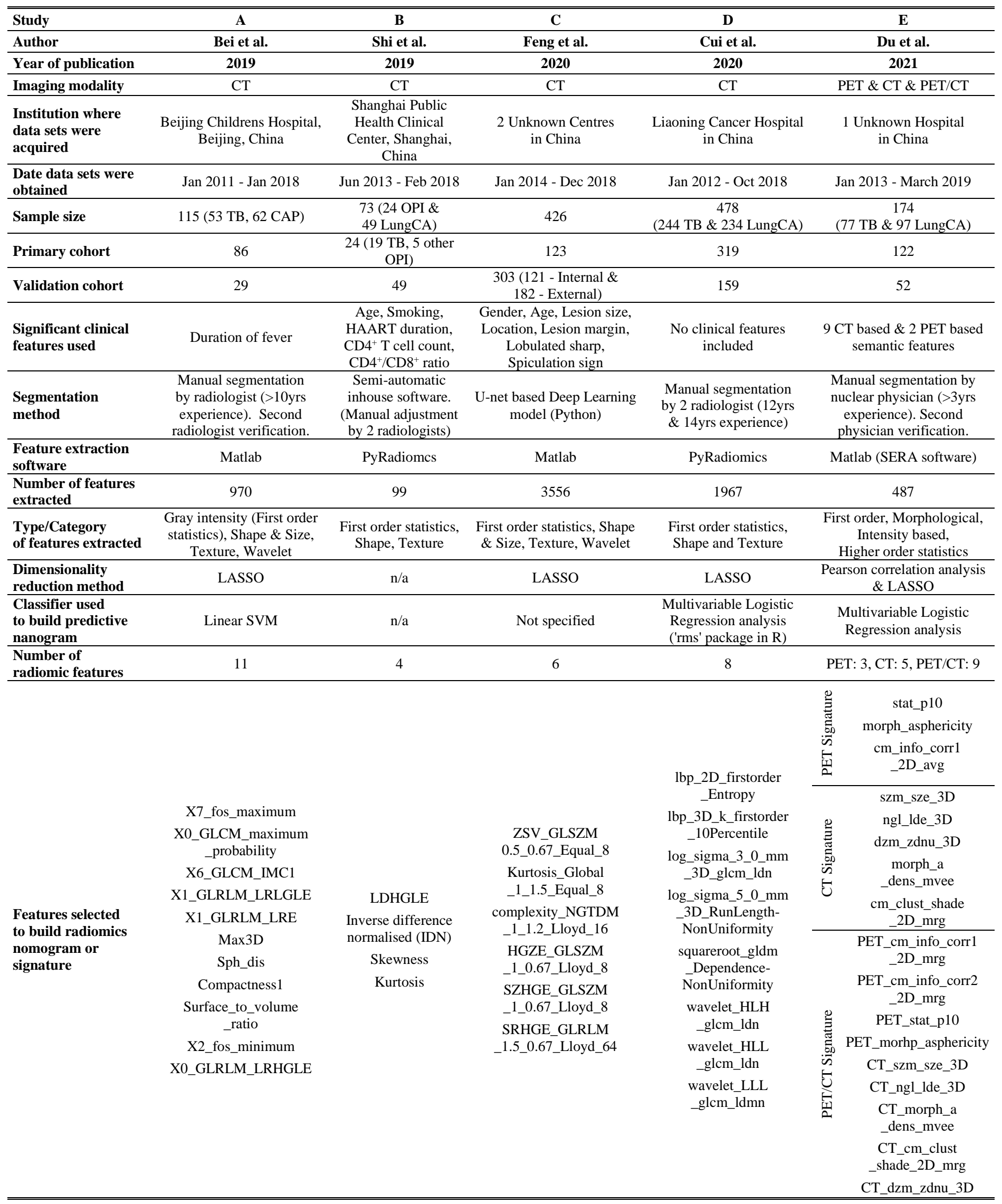

Abbreviations: $T B=$ Tuberculosis, CAP = Community Acquired Pneumonia, OPI = Opportunistic Pulmonary Infections, LungCA = Lung Cancer, HAART = Highly Active Antiretroviral Therapy, LASSO = Least absolute shrinkage and selection operator, SVM = Support Vector Machine, GLCM = Gray Level Cooccurrence Matrix, GLSZM = Gray Level Size Zone Matrix, GLRLM = Gray Level Run Length Matrix, GLDM = Gray Level Dependence Matrix, NGTDM = Neighboring Gray Tone Difference Matrix, LDHGLE = Large dependence high gray level emphasis 
Table 2. Summary of radiomic features used in each reviewed study.

\begin{tabular}{|c|c|c|c|c|c|c|}
\hline \multirow{2}{*}{\multicolumn{2}{|c|}{ Study }} & $\mathbf{A}$ & B & $\mathrm{C}$ & D & $\mathbf{E}^{*}$ \\
\hline & & Bei et al. & Shi et al. & Feng et al. & Cui et al. & Du et al. \\
\hline \multicolumn{2}{|c|}{$\begin{array}{l}\text { 1st order } \\
\text { features }\end{array}$} & $\begin{array}{l}\text { X7_fos_maximum } \\
\text { X2_fos_minimum }\end{array}$ & $\begin{array}{l}\text { Skewness } \\
\text { Kurtosis }\end{array}$ & $\begin{array}{l}\text { Kurtosis_Global } \\
\text {-1_1.5_Equal_8 }\end{array}$ & $\begin{array}{c}\text { lbp_2D_firstorder } \\
\text { _Entropy } \\
\text { lbp_3D_k_firstorder } \\
\text { _10Percentile }\end{array}$ & PET_stat_p10 \\
\hline \multicolumn{2}{|c|}{ Shape } & $\begin{array}{c}\text { Max3D } \\
\text { Sph_dis } \\
\text { Compactness1 } \\
\text { Surface_to_volume_ratio }\end{array}$ & --- & --- & $\begin{array}{l}\text { log_sigma_3_0_mm } \\
\text { _3D_glcm_ldn } \\
\text { log_sigma_5_0_mm } \\
\text { 3D_RunLength- } \\
\text { NonUniformity }\end{array}$ & $\begin{array}{l}\text { PET_morhp_asphericity } \\
\text { CT_morph_a_dens_mvee }\end{array}$ \\
\hline \multirow{7}{*}{ 茪 } & GLCM & $\begin{array}{c}\text { X0_GLCM } \\
\text { _maximum_probability } \\
\text { X6_GLCM_IMC1 }\end{array}$ & $\begin{array}{l}\text { Inverse difference } \\
\text { normalized (IDN) }\end{array}$ & --- & $\begin{array}{l}\text { wavelet_HLH } \\
\text { _glcm_ldn } \\
\text { wavelet_HLL } \\
\text { _glcm_ldn } \\
\text { wavelet_LLL } \\
\text { _glcm_ldmn }\end{array}$ & $\begin{array}{l}\text { CT_cm_clust } \\
\text { shade_2D_mrg } \\
\text { PET_cm_info } \\
\text { _corr1_2D_mrg } \\
\text { PET_cm_info } \\
\text { _corr2_2D_mrg }\end{array}$ \\
\hline & GLRLM & $\begin{array}{c}\text { X1_GLRLM_LRE } \\
\text { X0_GLRLM_LRHGLE } \\
\text { X1_GLRLM_LRLGLE }\end{array}$ & --- & $\begin{array}{l}\text { SRHGE_GLRLM } \\
\text {-1.5_0.67_Lloyd_64 }\end{array}$ & --- & --- \\
\hline & GLDM & --- & LDHGLE & --- & $\begin{array}{l}\text { squareroot_gldm } \\
\text { _Dependence- } \\
\text { NonUniformity }\end{array}$ & --- \\
\hline & GLSZM & --- & --- & $\begin{array}{c}\text { HGZE_GLSZM } \\
\text { _1_0.67_Lloyd_8 } \\
\text { SZHGE_GLSZM } \\
\text {-1_0.67_Lloyd_8 } \\
\text { ZSV_GLSZM } \\
\text {-0.5_0.67_Equal_8 }\end{array}$ & --- & CT_szm_sze_3D \\
\hline & GLDZM & --- & --- & --- & --- & CT_dzm_zdnu_3D \\
\hline & NGTDM & --- & --- & $\begin{array}{c}\text { complexity_NGTDM } \\
\text { _1_1.2_Lloyd_16 }\end{array}$ & --- & --- \\
\hline & NGLDM & --- & --- & --- & --- & CT_ngl_lde_3D \\
\hline
\end{tabular}

Abbreviations: GLCM = Gray Level Cooccurrence Matrix, GLRLM = Gray Level Run Length Matrix, GLDM = Gray Level Dependence Matrix, GLSZM = Gray Level Size Zone Matrix, GLDZM = Gray Level Distance Zone Matrix, NGTDM = Neighboring Gray Tone Difference Matrix, NGLDM = Neighboring Grey Level

* Since the PET/CT signature is simply a combination of the CT and PET signatures, only the PET/CT features are listed to avoid duplication.

Four of the reviewed studies ${ }^{\mathbf{2 9 , 3 3 - 3 5}}$ built radiomics nomograms, while one study ${ }^{32}$ only developed a radiomics signature. Table 2 is a summary of the significant radiomic features organised according to the feature groups. There was a minimal correlation between the features selected as significant in the five studies. The only two features that were identified by more than one study to be significant were Kurtosis ${ }^{32-33}$ and $10^{\text {th }}$ Percentile $^{\text {34-35 }}$. Kurtosis is a measure of the sharpness of the peak of the distribution of the values in the region of interest, ${ }^{28}$ and $10^{\text {th }}$ percentile of the $\mathrm{N}^{\text {th }}$ voxel in the ROI is a first-order statistical feature. ${ }^{28}$ Not one study identified any higher-order statistical features as significant.

There were some weaknesses noticed in the articles reviewed. The training and validation cohorts of most studies (all except for study $\mathrm{C}$ which had both an internal and external validation cohort) were from the same centers. However, it is preferable to have external validation cohorts when DL methods are used to reveal the possible overfitting of the training data sets. All studies were also retrospective studies, which is the only way to obtain a reasonable sample size in a new field of study. But the downside to this is that the researchers had no control over the acquisition parameters and the management of the data sets. Unfortunately, acquisition and post-processing play a vital role in high throughput quantitative image analysis.

The studies identified between 4 and 11 (mean $7.6 \pm 2.4$ ) radiomics features as significant from 99 to 3556 (mean 1415.8 \pm 1239.3 ) features extracted..$^{\mathbf{2 9 , 3 2 - 3 3}}$ Small data sets are generally a limitation of radiomic studies. Thousands of radiomic features are available for extraction, but datasets are often smaller than the number of possible features to mine. This was also the case in the reviewed articles where the primary cohort sample sizes ranged from 24 to 319 (mean $134.8 \pm 98.9$ ) patients, while the number of features extracted ranged from 99 to 3556 (mean $1415.8 \pm 1239.3){ }^{32-34,36}$ The number of features extracted is almost ten times more than the number of patients. A prospective multicenter study with larger cohorts is necessary to confirm the 
results and improve the reliability of all studies. This suggestion was also acknowledged by most authors from the reviewed studies. From the limited number of studies available no preliminary conclusions can be made regarding which features or feature groups are likely to produce robust and reliable quantitative image information from PTB radiological images.

Radiomics is intrinsically a complex multi-step process, but to further complicate this is a lack of standardised guidelines and definitions. A lack of consensus on general definitions makes study intercomparison difficult. Since reproducibility and robustness are vital in radiomics studies, all image processing and feature extraction details should be clearly recorded and disclosed. The Image Biomarker Standardization Initiative (IBSI) was published in 2019 and hopes to address various issues that will improve the validation and reproducibility of radiomics studies. ${ }^{37}$ All five of the reviewed articles did well to comply with these guidelines, with the most recent ${ }^{35}$ conforming the best.

The low number of studies included in this review article might seem like unconvincing evidence to address the purpose of this study, but this does indicate two things. Firstly, highthroughput quantitative image analysis, especially in nonneoplastic deceases, is a new but rapidly growing field of study. ${ }^{37}$ This is evident from the publication dates of the 5 articles that qualified to be included in this review study (2019 to 2021). Secondly, it shows that the possibility of using radiomics for quantitative image analysis for the purpose of PTB disease management and differentiation from other pulmonary diseases is still an under-investigated field of study.

\section{Conclusions}

Six data bases were searched and only five articles could be found detailing the application of radiomics to pulmonary tuberculosis. In all five articles reviewed, radiomic feature extractions from CT or PET/CT images were used effectively to quantify digital medical images for the purpose of comparing or differentiating tuberculosis from another pulmonary disease.

The outcome of this study evidently raises two questions.

1. Firstly, why has no attempt yet been made to use radiomics for the quantitative management and prognosis of pulmonary tuberculosis? Particularly while researchers still have a major challenge to identify more sensitive and specific biomarkers that can be used to identify the different stages of tuberculosis and to quantitively evaluate disease progression or response to treatment.

2. Secondly, why are all current radiomics studies on pulmonary tuberculosis performed from three-dimensional imaging modalities (CT or PET/CT scans) when patients in countries where pulmonary tuberculosis is most prevalent have very limited access to these modalities?

It can be concluded from this review that the application of radiomics feature extraction and analysis of pulmonary tuberculosis, for the purpose of quantitative decease management and prognostication from chest $\mathrm{x}$-rays, is a valuable data mining opportunity yet to be realised. This might seem challenging at this point, but with the fast development of computer science and the creative application of established mathematical solutions, this might be realised in the near future.

\section{References}

1. WHO. Global tuberculosis report 2020. Report. Geneva: World Health Organization, 2020.

2. Prevention CfDCa [Internet]. Testing for tuberculosis (tb).

Available from: https://www.cdc.gov/tb/publications/factsheets/testing/tb_testing.htm

3. Tan JH, Acharya UR, Tan C, Abraham KT, Lim CM. Computer-assisted diagnosis of tuberculosis: A first order statistical approach to chest radiograph. Journal of Medical Systems. 2012; 36(5):2751-9. https://doi.org/10.1007/s10916-011-9751-9

4. Lewinsohn DM, Leonard MK, LoBue PA, Cohn DL, Daley CL, Desmond E, et al. Official american thoracic society/infectious diseases society of america/centers for disease control and prevention clinical practice guidelines: Diagnosis of tuberculosis in adults and children. Clinical infectious diseases: an official publication of the Infectious Diseases Society of America. 2017; 64(2):111-5. https://doi.org/10.1093/cid/ciw778

5. Chen RY, Dodd LE, Lee M, Paripati P, Hammoud DA, Mountz JM, et al. Pet/ct imaging correlates with treatment outcome in patients with multidrug-resistant tuberculosis. Science translational medicine. 2014;6(265):265ra166.

https://doi.org/10.1126/scitranslmed.3009501

6. Drain PK, Gardiner J, Hannah H, Broger T, Dheda K, Fielding K, et al. Guidance for studies evaluating the accuracy of biomarkerbased nonsputum tests to diagnose tuberculosis. Journal of Infectious Diseases. 2019;220:S108-S115. https://doi.org/10.1093/infdis/jiz356

7. Goletti D, Petruccioli E, Joosten SA, Ottenhoff TH. Tuberculosis Biomarkers: From Diagnosis to Protection. Infect Dis Rep. 201624;8(2):6568. https://doi.org/10.4081/idr.2016.6568 
8. Melendez J, Ginneken Bv, Maduskar P, Philipsen RHHM, Ayles H, Sánchez CI. On combining multiple-instance learning and active learning for computer-aided detection of tuberculosis. IEEE Transactions on Medical Imaging. 2016;35(4):1013-24. https://doi.org/10.1109/TMI.2015.2505672

9. Santosh KC, Antani S. Automated chest x-ray screening: Can lung region symmetry help detect pulmonary abnormalities? IEEE transactions on medical imaging. 2018;37(5):1168-77. https://doi.org/10.1109/TMI.2017.2775636

10. Skoura E, Zumla A, Bomanji J. Imaging in tuberculosis. International Journal of Infectious Diseases. 2015;32:87-93. https://doi.org/10.1016/j.ijid.2014.12.007

11. Chassagnon G, Vakalopoulou M, Paragios N, Revel MP. Artificial intelligence applications for thoracic imaging. European Journal of Radiology. 2020;123:108774. https://doi.org/10.1016/j.ejrad.2019.108774

12. Mettler FA, Jr., Huda W, Yoshizumi TT, Mahesh M. Effective doses in radiology and diagnostic nuclear medicine: A catalog. Radiology. 2008; 248(1):254-63. https://doi.org/10.1148/radiol.2481071451

13. Van't Hoog AH, Meme HK, van Deutekom H, et al. High sensitivity of chest radiograph reading by clinical officers in a tuberculosis prevalence survey. Int J Tuberc Lung Dis. 2011;15(10):1308-14. https://doi.org/10.5588/ijtld.11.0004.

14. Lambin P, Leijenaar RTH, Deist TM, Peerlings J, de Jong EEC, et al. Radiomics: The bridge between medical imaging and personalized medicine. Nat Rev Clin Oncol. 2017;14(12):749-762. https://doi.org/10.1038/nrclinonc.2017.141

15. Hogeweg L, Sánchez CI, Maduskar P, Philipsen R, Story A, Dawson R, et al. Automatic detection of tuberculosis in chest radiographs using a combination of textural, focal, and shape abnormality analysis. IEEE Transactions on Medical Imaging. 2015;34(12):2429-42. https://doi.org/10.1109/TMI.2015.2405761

16. Ginneken Bv, Katsuragawa S, ter Haar Romeny, Kunio D, Viergever MA. Automatic detection of abnormalities in chest radiographs using local texture analysis. IEEE Transactions on Medical Imaging. 2002;21(2):139-49. https://doi.org/10.1109/42.993132

17. Shen R, Cheng I, Basu A. A hybrid knowledge-guided detection technique for screening of infectious pulmonary tuberculosis from chest radiographs. IEEE Transactions on Biomedical Engineering. 2010;57(11):2646-56. https://doi.org/10.1109/TBME.2010.2057509

18. Melendez J, Ginneken Bv, Maduskar P, Philipsen RHHM, Reither K, Breuninger M, et al. A novel multiple-instance learning-based approach to computer-aided detection of tuberculosis on chest X-rays. IEEE Transactions on Medical Imaging. 2015;34(1):179-92. https://doi.org/10.1109/TMI.2014.2350539

19. Jaeger S, Juarez-Espinosa OH, Candemir S, Poostchi M, Yang F, Kim L, et al. Detecting drug-resistant tuberculosis in chest radiographs. International journal of computer assisted radiology and surgery. 2018;13(12):1915-25. https://doi.org/10.1007/s11548018-1857-9

20. Abideen ZU, Ghafoor M, Munir K, Saqib M, Ullah A, Zia T, et al. Uncertainty assisted robust tuberculosis identification with bayesian convolutional neural networks. IEEE Access. 2020;8:22812-25. https://doi.org/10.1109/ACCESS.2020.2970023

21. Summers RM. Are we at a crossroads or a plateau? Radiomics and machine learning in abdominal oncology imaging. Abdominal Radiology. 2019;44(6):1985-9. https://doi.org/10.1007/s00261-018-1613-1

22. Pesapane F, Codari M, Sardanelli F. Artificial intelligence in medical imaging: Threat or opportunity? Radiologists again at the forefront of innovation in medicine. European Radiology Experimental. 2018;2(1):1-10. https://doi.org/10.1186/s41747-018-0061-6

23. Rizzo S, Botta F, Raimondi S, Origgi D, Fanciullo C, Morganti AG, et al. Radiomics: The facts and the challenges of image analysis. European Radiology Experimental. 2018;2(1):1-8. https://doi.org/10.1186/s41747-018-0068-z

24. Gillies RJ, Kinahan PE, Hricak H. Radiomics: Images are more than pictures, they are data. Radiology. 2016; 278(2):563-77. https://doi.org/10.1148/radiol.2015151169

25. Kumar V, Gu Y, Basu S, Berglund A, Eschrich SA, Schabath MB, et al. Radiomics: The process and the challenges. Magnetic resonance imaging. 2012;30(9):1234-48. https://doi.org/10.1016/j.mri.2012.06.010

26. Papanikolaou N, Matos C, Koh DM. How to develop a meaningful radiomic signature for clinical use in oncologic patients. Cancer Imaging. 2020;20(1):33. https://doi.org/10.1186/s40644-020-00311-4

27. Liu Q, Li J, Liu F, Yang W, Ding J, Chen W, et al. A radiomics nomogram for the prediction of overall survival in patients with hepatocellular carcinoma after hepatectomy. Cancer Imaging. 2020;20(1):82. https://doi.org/10.1186/s40644-020-00360-9

28. van Griethuysen JJM, Fedorov A, Parmar C, Hosny A, Aucoin N, Narayan V, et al. Computational radiomics system to decode the radiographic phenotype. Cancer research. 2017;77(21):e104-e7. https://doi.org/10.1158/0008-5472.CAN-17-0339

29. Bei W, Min L, He M, Fangfang H, Yan W, Shunying Z, et al. Computed tomography-based predictive nomogram for differentiating primary progressive pulmonary tuberculosis from community-acquired pneumonia in children. BMC Medical Imaging. 2019;19:63. https://doi.org/10.1186/s12880-019-0355-z

30. Lambin P, Rios-Velazquez E, Leijenaar R, Carvalho S, van Stiphout RGPM, Granton P, et al. Radiomics: Extracting more information from medical images using advanced feature analysis. European Journal of Cancer. 2012;48(4):441-6. https://doi.org/10.1016/j.ejca.2011.11.036 
31. Moher D, Liberati A, Tetzlaff J, Altman DG, The PG. Preferred reporting items for systematic reviews and meta-analyses: The prisma statement. PLOS Medicine. 2009;6(7):e1000097. https://doi.org/10.1371/journal.pmed.1000097

32. Shi W, Zhou L, Peng X, Ren H, Wang Q, Shan F, et al. Hiv-infected patients with opportunistic pulmonary infections misdiagnosed as lung cancers: The clinicoradiologic features and initial application of ct radiomics. Journal of thoracic disease. 2019;11(6):2274-86. https://doi.org/10.21037/jtd.2019.06.22

33. Feng B, Chen X, Chen Y, Liu K, Li K, Liu X, et al. Radiomics nomogram for preoperative differentiation of lung tuberculoma from adenocarcinoma in solitary pulmonary solid nodule. European Journal of Radiology. $2020 ; 128$. https://doi.org/10.1016/j.ejrad.2020.109022

34. Cui EN, Yu T, Shang S-J, Wang X-Y, Jin Y-L, Dong Y, et al. Radiomics model for distinguishing tuberculosis and lung cancer on computed tomography scans. World Journal of Clinical Cases. 2020;8(21):5203-12. https://doi.org/10.12998/wjcc.v8.i21.5203

35. Du D, Gu J, Chen X, Lv W, Feng Q, Rahmim A, et al. Integration of pet/ct radiomics and semantic features for differentiation between active pulmonary tuberculosis and lung cancer. Molecular Imaging \& Biology. 2021;23(2):287-298. https://doi.org/10.1007/s11307020-01550-4

36. Cui EN, Yu T, Shang SJ, Wang XY, Jin YL, Dong Y, et al. Radiomics model for distinguishing tuberculosis and lung cancer on computed tomography scans. World journal of clinical cases. 2020;8(21):5203-12. https://doi.org/10.12998/wjcc.v8.i21.5203

37. Zwanenburg A, Vallières M, Abdalah MA, Aerts HJWL, Andrearczyk V, Apte A, et al. The image biomarker standardisation initiative: Standardised quantitative radiomics for high-throughput image-based phenotyping. Radiology. 2020;295(2):328-38. https://doi.org/10.1148/radiol.2020191145 